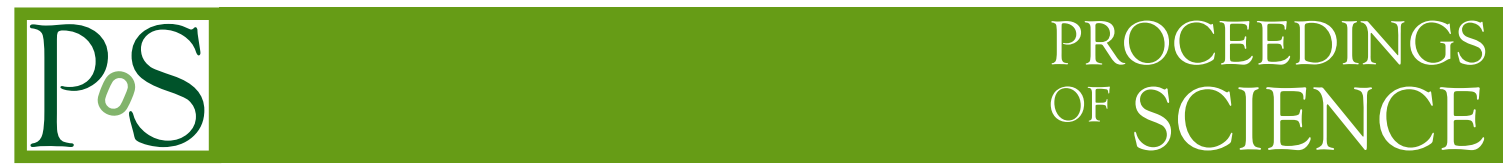

\title{
Reusing KTeV CsI crystals for J-PARC KOTO experiment
}

\section{Yoko Yanagida*}

Osaka University

E-mail: yoko@champ.hep.sci.osaka-u.ac.jp

Hiroki Yoshimoto

Osaka University

E-mail: hirokiechamp.hep.sci.osaka-u.ac.jp

The $\mathrm{K}^{0} \mathrm{TO}$ experiment will reuse CsI crystals which were used at Fermilab KTeV experiment to improve the position and energy resolutions. We will describe how we unstacked and shipped a total of 2790 CsI crystals in 2008.

2009 KAON International Conference

June 09 - 12, 2009

Tsukuba, Japan

${ }^{*}$ Speaker. 


\section{Introduction}

The $\mathrm{K}^{0} \mathrm{TO}$ experiment at the J-PARC is searching for the $K_{L} \rightarrow \pi^{0} v \bar{v}$ decay. The branching ratio of this decay directly measures the parameter $\eta$ of the CKM matrix. If the measured branching ratio is different from the standard model prediction, it means there is new physics beyond the Standard Model that violates CP (see Nanjo's paper for more details).

The $K_{L} \rightarrow \pi^{0} v \bar{v}$ decay can be measured by finding two photons from the $\pi^{0}$. In order to measure the energies and positions of the photons, we will use pure CsI crystals for calorimeter. The pilot experiment of $\mathrm{K}^{0} \mathrm{TO}$, the E391a experiment at KEK used $7 \times 7 \times 30 \mathrm{~cm}^{3}$ pure CsI crystals.

The $\mathrm{K}^{0} \mathrm{TO}$ experiment will replace the E391a crystals with pure CsI crystals and PMTs which were used for FNAL KTeV experiment to improve position and energy resolutions. The position resolution will be improved mainly because the crystal cross sections are smaller $\left(2.5 \times 2.5 \mathrm{~cm}^{2}\right.$ and $5 \times 5 \mathrm{~cm}^{2}$ ) as shown in Fig. 11 The energy resolution will be improved because of their longer length, $50 \mathrm{~cm}\left(27 \mathrm{X}_{0}\right)$, compared to $30 \mathrm{~cm}\left(16 \mathrm{X}_{0}\right)$ of the E391a crystals. The amount of shower leakage will be reduced as shown in Fig. 2 .

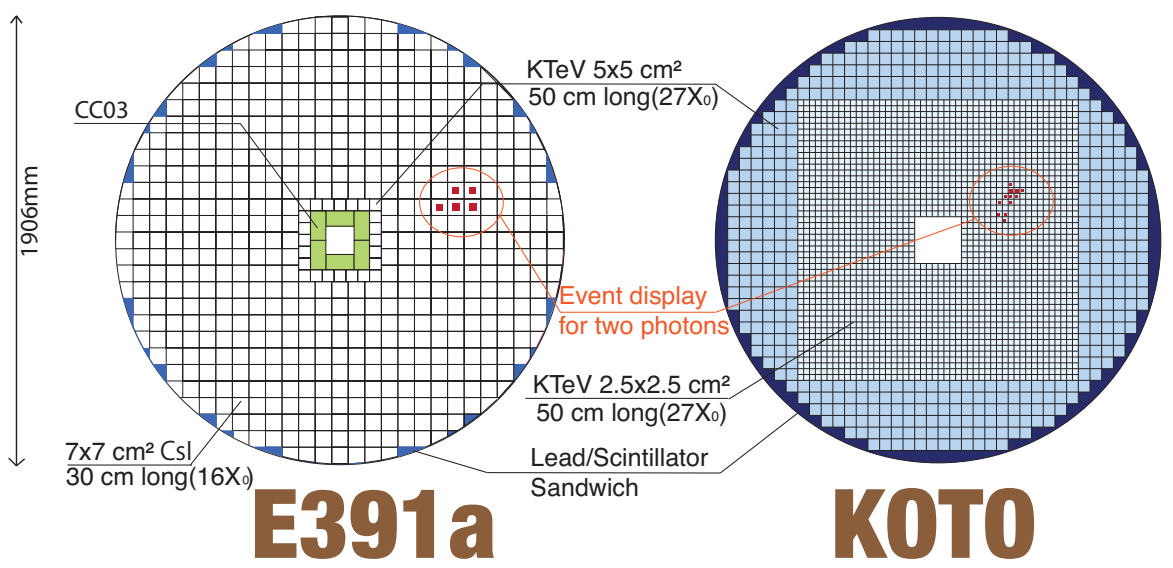

Figure 1: Front views of the E391a CsI calorimeter (left) and $\mathrm{K}^{0} \mathrm{TO}$ CsI calorimeter (right).

We will describe how we prepared and shipped the CsI crystals from Fermilab to Osaka University.

\section{Unstacking and packing all of the KTeV CsI in 2008}

The KTeV calorimeter had 2232 small crystals and 868 large crystals. Of them, all the small crystals and 36 small spare crystals, and 530 large crystals were shipped to Osaka University. Other 307 large crystals were shipped to Chicago University. For this project, students in the $\mathrm{K}^{0} \mathrm{TO}$ experiment traveled to FNAL 8 times in 2008.

Figure 5 shows one crystal and its readout materials etc.. Small crystals and large crystals have the same structure. Each CsI crystal is wrapped with a partially aluminized mylar with a black paint coated on the outside. The scintillation light from the CsI crystal is read out by a phototube. In 


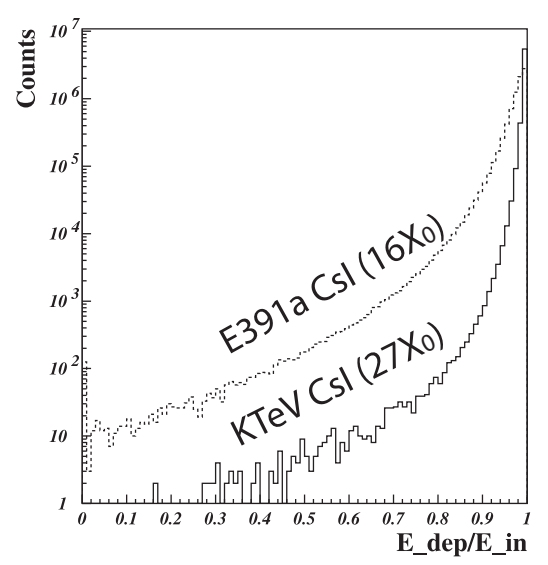

Figure 2: Monte Carlo study of the shower leakage. The ratio between the deposited energy in the CsI crystals and the true incident energy for the photons in the energies between $0.1 \mathrm{GeV}$ and $1 \mathrm{GeV}$.

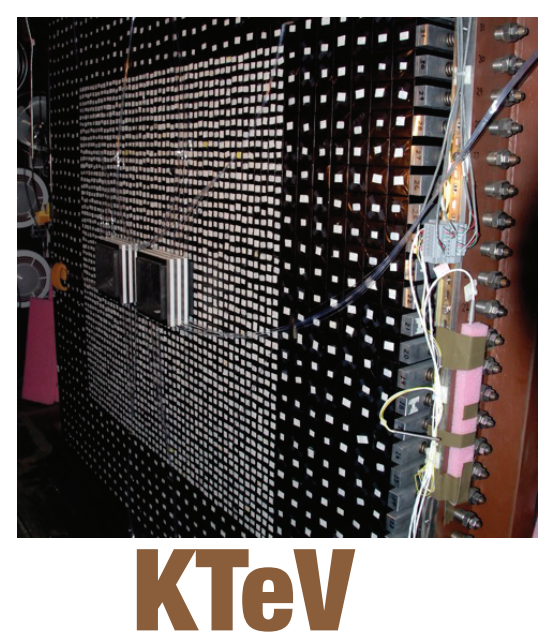

Figure 3: KTeV CsI calorimeter

order to hold each phototube and shield it from magnetic field, a mu-metal pipe with a square supporting block (which we call flange) made of Delrin are glued on to the crystal. The flange also has one small hole ( $2 \mathrm{~mm} \phi$ ) to insert optical fiber for calibration.

\subsection{Unstacking and packing}

The work for unstacking and packing crystals was done by three persons.

First, each crystal was placed on a glass plate to keep it flat. A fiber holder, the hooks and HV divider were removed. The crystal, PMT, and the glass plate were placed inside a moisture barrier pack and vacuum-sealed. The sealed package was covered with two Al angles with rubber cushions inside for mechanical protection. At the end of the day, the packed crystals were brought out from the CsI room and received a radiation check. Then the packed crystals were installed in air freight cases. 


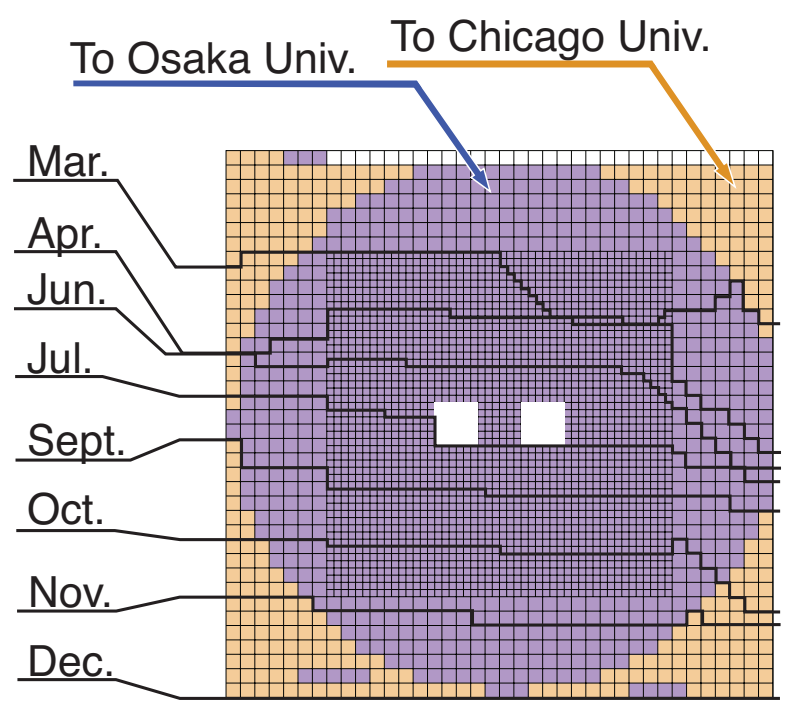

Figure 4: Shipping in 2008, viewed from upstream

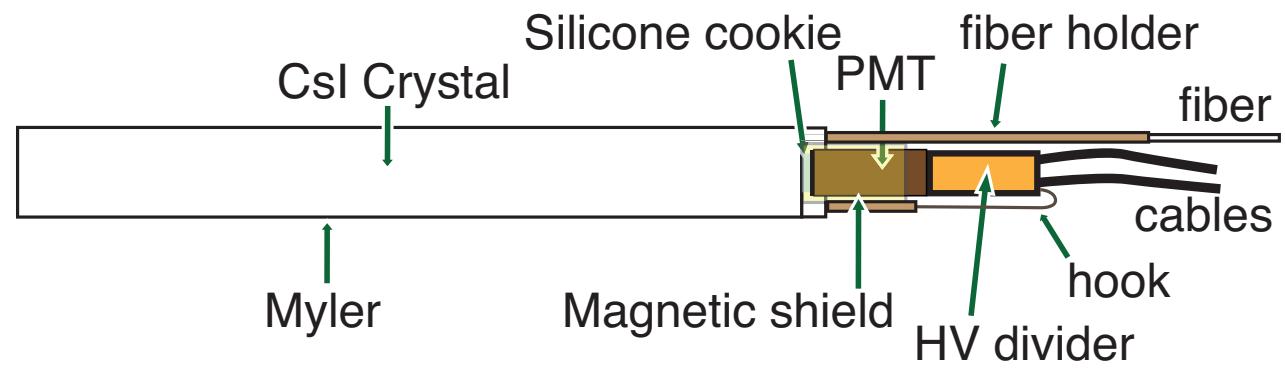

Figure 5: One crystals and its readout system etc..

Three persons worked 7 hours a day with occasional breaks, and 6 days per visit. In each visit, $8 \sim 12$ crates ( $200 \sim 450$ blocks ) were packed and shipped.

At Osaka University, crystals were brought into a dry storage room with a relative humidity of less than $10 \%$. The crystals were removed from the $\mathrm{Al}$ angles and stacked in a tray which can be hold 24 small crystals or 8 large crystals.

\section{Difference between $\mathrm{KTeV}$ and $\mathrm{K}^{0} \mathrm{TO}$ CsI calorimeters and our modification}

There are several differences between $\mathrm{KTeV}$ and $\mathrm{K}^{0} \mathrm{TO}$ CsI calorimeters.

- Environment

At the $\mathrm{K}^{0} \mathrm{TO}$ experiment, the CsI crystals are placed in vacuum, instead of a dry room, and it is not easy to access the calorimeter during the experiment. We thus need a reliable structure to hold PMTs and their bases. In order to reduce the amount of heat produced in vacuum, we 
will use low power Cockcroft-Walton bases for PMTs. We are now checking the performance of the PMTs and the other read out materials to work in vacuum.

- The overall shape

The crystals will be stacked inside a cylinder as shown in Fig. 11 We will fill the gap between the CsI crystals and the cylinder with Lead/scintillator sandwich detector modules.

- Connection between CsI crystal and PMT

Although we could reglue crystals and flanges, the flanges could drop off. Thus we will make one large Al or stainless steel plate to hold all the PMTs in place.

\section{Conclusion}

In 2008, we finished transferring all the CsI crystals that we need for $\mathrm{K}^{0} \mathrm{TO}$ experiment to Japan. The crystals are kept in a dry room with a relative humidity of less than $10 \%$. We will test the uniformity and light yield of the crystals and performance of all PMTs. In the next spring, we will stack the crystals at J-PARC. The technical know-how that we established from this shipping will help us to install crystals.

\section{References}

[1] J. Comfort et al., [J-PARC E14 Collab.], "Proposal for $K_{L} \rightarrow \pi^{0} v \bar{v}$ Experiment at J-PARC" (2006), http://osksn2.hep.sci.osaka-u.ac.jp/ taku/jparcKL/jparc_E14_proposal.pdf 